cholangiopathy. $\left[{ }^{18} \mathrm{~F}\right]$-fluorodeoxyglucose positron emission tomography/computed tomography (FDG PET/CT) is the only technique that allows imaging of metabolic activity by detecting FDG accumulation in cells and correlation with anatomical structures. Increased tracer uptake is typically seen in inflammatory or neoplastic tissue thereby potentially aiding diagnosis, and assessment of disease extent and activity. There is limited data currently available on its utility in IgG4-RD and whether this varies according to presentation. The aim of this study is to determine the utility of FDG $\mathrm{PET} / \mathrm{CT}$ in diagnosis, monitoring disease activity and identifying multi system involvement.

Methods We performed a retrospective study of a prospectively maintained multi-disciplinary IgG4-RD database to identify patients who underwent FDG PET/CT over a 3year period. Additional organ involvement and change in management consequent on FDG PET/CT was recorded. Fisher's exact test was used for the comparison of proportions.

Results 25 patients with a diagnosis or suspicion of IgG4RD underwent FDG PET/CT between November 2016 and October 2019. The median age [IQR] at presentation was 59 [48.5-65.5], $18(72 \%)$ were male. 15 (72.5\%) suspected or proven $\mathrm{PB}$ disease, $6(24 \%)$ head and neck (HN), 1 (4\%) each of retroperitoneal, both $\mathrm{PB}$ and $\mathrm{HN}$, pulmonary and renal. In $22(88 \%)$ cases $(15 / 15 \mathrm{~PB}, 7 / 10$ non PB) FDG $\mathrm{PET} / \mathrm{CT}$ findings had a direct impact on management. The difference in utility between PB (100\%) and non-PB (70\%) was not quite statistically significant $(\mathrm{p}=0.059)$. In 1 patient it enabled exclusion of PB IgG4-RD. In 15 (60\%) it led to a decision to escalate therapy this included 3 AIP cases (21.4\% of definite PB cases) in which new organ involvement was identified. In 6 cases (5 PB and 1 renal IgG4-RD) with concern of active disease because of persistently elevated or rising IgG4 levels it excluded FDG avid inflammation.

Conclusion In this retrospective study FDG PET/CT had a clinically important impact on management of IgG4-RD. Identifying other organ involvement as well as influencing therapeutic decision making particularly in PB disease. Further studies are required to fully delineate its role in IgG4-RD.

\section{P252 URINARY VOLATILE ORGANIC COMPOUNDS AS A BIOMARKER FOR PANCREATIC CANCER}

James Baker*, Prashant Aggarwal*, Elinor Chapman, Eithne Costello-Goldring, Bill Greenhalf, Chris Probert. University of Liverpool, Liverpool, UK; *JP and PA contributed equally and share first authorship*

\subsection{6/gutjnl-2020-bsgcampus.326}

Introduction Pancreatic cancer is the 5th commonest cause of cancer mortality in the UK, with ca. 10,000 new cases of pancreatic cancer annually. Late diagnosis results in poor survival: $80 \%$ of cases present late. Volatile organic compounds (VOCs) have been investigated as biomarkers for many disorders. We report the first pilot study of urinary VOC biomarkers for pancreatic cancer using gas chromatography-mass spectrometry (GC-MS) analysis.

Method Urine was collected from patients with pancreatic cancer $(n=4$; mean age 66 ys, range 43-82 ys), lung cancer $(\mathrm{n}=17$; mean age $61 \mathrm{ys}$, range $48-74 \mathrm{ys})$, and healthy controls $(n=6$; mean age 31 , range 22-57). All samples were stored at $-80^{\circ} \mathrm{C}$. Later they were thawed, and $0.5 \mathrm{ml}$ aliquots were placed in $10 \mathrm{ml}$ headspace vials and either $0.1 \mathrm{ml}$ of sodium hydroxide or sulphuric acid solution were added. GCMS was performed. A library of VOCs was built using AMDIS and NIST software. We used the $\mathrm{R}$ package Metab and MetaboAnalyst software for data analysis. P values were reported based on t-tests, boxplots of fold change of potential biomarkers are shown (figure 1): compounds have been coded until IP is protected.

Results Acidified Results

2 compounds (A\&B) were significantly more abundant in pancreatic cancer compared to lung cancer $(p<0.0001 \&$ $\mathrm{p}=0.008$, respectively) and also vs controls $(\mathrm{p}=0.006 \&$ $\mathrm{p}=0.002$, respectively); a $3 \mathrm{rd}$ compound $(\mathrm{C})$ was reduced in pancreatic cancer compared to lung cancer $(p=0.021)$; and 6 additional compounds were significantly more abundant in pancreatic cancer compared to healthy controls $(\mathrm{p}<0.05$; range: $\mathrm{p}=0.001-0.047)$.

After corrections for multiple comparison, 1 compound (A) remains raised with pancreatic cancer compared to lung cancer $(\mathrm{FDR}=0.001) . \mathrm{Q}^{2}$, a performance marker of the PLS-DA model, is 0.5 suggesting a good predictor of group membership and no overfitting of data.

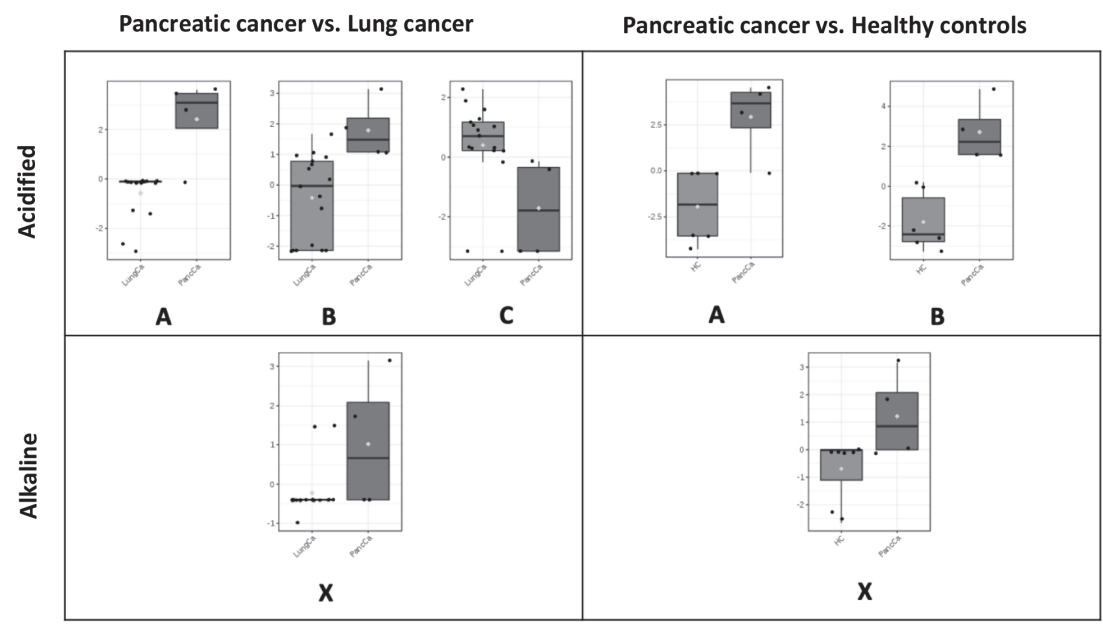

Abstract 252 Figure 1 Boxplots to show fold change of potential biomarkers for pancreatic cancer Key: LungCa=Lung cancer, PancCa=Pancreatic cancer, and $\mathrm{HC}=$ Healthy controls Letters $A, B, C$, and $X$ denote coded compounds 


\section{Alkaline Results}

1 compound (X) was significantly more abundant in pancreatic cancer compared to lung cancer $(p=0.020)$ and also vs controls $(p=0.045)$.

Conclusion This small pilot study is proof of concept indicating that urinary VOCs have potential as biomarkers for pancreatic cancer. 2 compounds $(\mathrm{A} \& \mathrm{~B})$ in pancreatic cancer are raised compared to lung cancer and healthy controls when urine is acidified and another (X) under alkaline conditions, of these 1 compound (A) remains raised after corrections of multiple comparison. Further work is required to develop urinary VOCs as biomarkers for pancreatic cancer.

\section{P253 FIRST UK REAL-WORD DATA ON PATIENTS WITH CARCINOID SYNDROME ON LONG-TERM TELOTRISTAT THERAPY}

Shweta Hota*, Elmie Cananea, Wendy Martin, Dominique Clement, Bernadette Solis, John Ramage, Rajaventhan Srirajaskanthan. Kings College Hospital, London, UK

\subsection{6/gutjnl-2020-bsgcampus.327}

Introduction Telotristat ethyl is a tryptophan hydroxylase inhibitor, effective against symptoms of carcinoid syndrome refractory to standard somatostatin analogue therapy by directly inhibiting serotonin production. While clinical trials have established short-term efficacy of the drug, we report the first exploratory study of 15 patients with metastatic neuroendocrine tumours (NET) on long-term telotristat (median duration $=8$ months). This is a novel EMA-approved treatment, not yet approved by NICE guidelines. Patients received telotristat via a compassionate use programme. The primary outcome of this study was to determine biochemical and symptomatic improvement after initiating telotristat. The secondary outcome was to define the demographic of patients at King's College Hospital typically started on telotristat therapy. Methods We performed a retrospective chart-review study of 15 patients diagnosed with metastatic small bowel NET with symptoms of carcinoid syndrome. Medical notes and clinic letters were reviewed for patient-reported symptoms, biochemical marker levels and imaging results. Stratified analysis was performed using the Wilcoxon sign-rank test.

Results All 15 patients initiated on telotristat, had a small bowel primary NET with metastatic disease ( 15 liver, 5 bone, 2 peritoneum). 8 patients had carcinoid heart disease, 7 having had previous valve surgery. 12 patients had completed Peptide Receptor Radionucleotide Therapy prior to initiating telotristat and 4 patients had Selective Internal Radiation Therapy prior to initiating telotristat. All patients were taking somatostatin analogue therapy. All patients showed significant reduction in urinary 5-HIAA (median percentage change $57.14 \%, \mathrm{p}=0.001)$. There was no significant change in chromogranin A or B. Of the 6 patients taking telotristat for at least 1-year, urinary 5-HIAA still remained significantly lower after 1-year (median percentage change 55.8\%, p = 0.028 ). Moreover, 9 patients reported improvement in diarrhoea, 5 reported improvement in cutaneous flushing, 4 reported weight stability. 4 patients reported side effects including abdominal pain and constipation. Only 2 patients showed progression of disease on imaging during the followup period.

Conclusion This is the first UK data on real-world use of this novel agent for carcinoid syndrome. While telotristat is currently only licensed for diarrhoea, patients on long-term telotristat also report improvement in flushing and weight loss, as well as significant persistent improvement in urinary 5-HIAA.

\section{P254 ARE WE STILL MISSING CASES OF PANCREATIC EXOCRINE INSUFFICIENCY AND PANCREATIC ATROPHY IN DIABETES MELLITUS?}

Mustafa Jalal*, Solomon Tesfaye, Andrew Hopper. Sheffield Teaching Hospital, Sheffield, UK

\subsection{6/gutjnl-2020-bsgcampus.328}

Introduction There is increasing evidence of coexistence of exocrine dysfunction in patients with diabetes mellitus (DM). Patients with pancreatic exocrine insufficiency (PEI) are at risk of malabsorption and malnutrition. In Leeds et al review there was a significant improvement of gastrointestinal symptoms and reduction of frequency of hypoglycaemia when treated with pancreatic enzyme replacement therapy (PERT). Our aim was to study the current practice and yield of PEI in DM patients tested with faecal elastase1 (FEL-1).

Methods Consecutive recruitment of DM patients attending diabetes outpatient clinic in a tertiary centre. FEL-1 $<200 \mu \mathrm{g} / \mathrm{g}$ considered PEI. Age, BMI, smoking history, alcohol intake, and duration of disease were collected. Those with PEI were followed up in our gastroenterology clinic.

Results 64 patients with DM were approached. Final analysis included 49 patients $(\mathrm{DM} 1=21, \mathrm{DM} 2=28)$ who returned stool sample, median age 62 years, 27 male (55.1\%). Ten patients (20.4\%) had low FEL-1 (DM1=5, DM2 =5). Six out of $10(60 \%)$ with low FEL-1 had morphological changes in the pancreas (chronic pancreatitis=1, pancreatic atrophy=5). Most patients did not have GI symptoms $(n=8)$ apart from diarrhoea $(n=1)$ and bloating $(\mathrm{n}=1)$. PEI patients had median BMI within overweight category $\left(28.5 \mathrm{~kg} / \mathrm{m}^{2}\right)$ compared to $\left(30.3 \mathrm{~kg} / \mathrm{m}^{2}\right)$ in normal FEL-1 group. Pack-year smoking history was higher in PEI group although didn't reach significance, 20 vs 12.5 , $p=0.7$. There was no difference in duration of disease between the two groups.

Conclusions Majority of our DM patients were asymptomatic however there was high prevalence of PEI and pancreatic atrophy. We expect higher prevalence of PEI in symptomatic DM patients attending gastroenterology clinic. Therefore, increased awareness and prompt screening can improve the diagnosis of PEI. Follow up study will be conducted to assess the impact of treatment on quality of life.

\section{P255 DIETARY NIACIN INTAKE IS INVERSELY ASSOCIATED WITH THE DEVELOPMENT OF GALLSTONES: A PROSPECTIVE COHORT STUDY}

${ }^{1}$ Hong Kai Lim*, ${ }^{1}$ Robert Luben, ${ }^{2}$ Andrew R Hart, ${ }^{3}$ Paul JR Banim. ${ }^{1}$ University of Cambridge, Cambridge, UK; ${ }^{2}$ University of East Anglia, Norwich, UK; ${ }^{3}$ James Paget University Hospital, Great Yarmouth, UK

\subsection{6/gutjnl-2020-bsgcampus.329}

Introduction Dietary niacin may prevent gallstones, a major cause of acute pancreatitis (AP), by increasing plasma high density lipoprotein and lowering triglycerides, both associated 